\title{
Polimorfismos Genéticos: Implicações na Patogênese do Carcinoma Medular de Tireóide
}

\begin{abstract}
RESUMO
O carcinoma medular de tireóide (CMT) é uma neoplasia maligna rara, ocorrendo na forma esporádica ou hereditária. Mutações germinativas no proto-oncogene RET são responsáveis pelo CMT hereditário. No entanto, a maioria dos casos de CMT ocorre em indivíduos sem história familiar, na qual a patogênese da doença ainda é pouco compreendida. Os polimorfismos do gene RET são descritos na população geral assim como em pacientes com CMT. Embora estas variações alélicas aparentemente não confiram qualquer atividade transformadora no receptor RET, estudos sugerem que essas alterações genéticas podem modificar a suscetibilidade à doença e o fenótipo clínico em pacientes com CMT esporádico ou hereditário. Uma maior freqüência dos polimorfismos localizados nos exons 11 (G691S), 13 (L769L), 14 (S836S) e 15 (S904) é descrita em pacientes com CMT provenientes de países americanos e europeus. Na presente revisão, analisamos criticamente os resultados obtidos nos diferentes estudos e descrevemos a freqüência dos polimorfismos do RET em pacientes brasileiros com CMT esporádico. (Arq Bras Endocrinol Metab 2007;51/5:723-730)
\end{abstract}

Descritores: Carcinoma medular de tireóide; Proto-oncogene RET; Polimorfismos; Patogênese

\section{ABSTRACT}

Genetic Polymorphisms: Implications in the Pathogenesis of Medullary Thyroid Carcinoma.

Medullary thyroid carcinoma (MTC) is a rare malignant neoplasia, which may occur on sporadic form or on a hereditary basis. Germ line mutations in the RET proto-oncogene is responsible for hereditary MTC. However, most MTC occur in individuals without family history where the pathogenesis is still unclear. Single nucleotide polymorphisms (SNPs) of the $R E T$ gene have been described in the general population as well as in patients with MTC. Even though these allelic variants do not seem to confer any transforming activity to the tyrosine kinase domain of the RET protein, cumulative studies suggest that they could modify disease susceptibility and clinical phenotype in patients with sporadic or hereditary MTC. Polymorphisms located in exons 11 (G691S), 13 (L769L), 14 (S836S), and 15 (S904S) seem to be over-represented in sporadic MTC patients from American and European countries. Here, we discuss the results obtained in different studies as well as describe the frequency of RET polymorphisms in Brazilian patients with sporadic MTC. (Arq Bras Endocrinol Metab 2007;51/5:723-730)

Keywords: Medullary thyroid carcinoma; RET proto-oncogene; Polymorphisms, Pathogenesis revisão

\author{
ANdReIa Possatti da Rocha \\ PATRÍCIA K. RIBEIRO MAGALHÃES \\ ANa LUIZA MaIA \\ LEA MARIA ZANINI MACIEL
}

Setor de Tireóide, Serviço de Endocrinologia, Hospital de Clínicas de Porto Alegre, Universidade Federal do Rio Grande do Sul (APR \& ALM), Porto Alegre, RS, e Divisão de Endocrinologia, Departamento de Clínica Médica, Faculdade de Medicina de Ribeirão Preto, Universidade de São Paulo (PKRM \& LMZM), Ribeirão Preto, SP.

Recebido em 19/01/07

Aceito em 23/01/07 
$\mathrm{D}$ ESDE A IDENTIFICAÇÃO DO PROTO-ONCOGENE RET como gene causador do carcinoma medular de tireóide (CMT) hereditário em 1993, o conhecimento acumulado sobre a patogênese do CMT e neoplasias associadas tem sido significativo (1-5). Contudo, particularidades da doença ainda são pouco compreendidas. Como exemplos, a heterogeneidade clínica observada em indivíduos com a mesma mutação e questões quanto à possibilidade da mutação no RET não ser o evento inicial na gênese da doença (68). Em relação ao CMT esporádico o quadro é um pouco mais obscuro. Mutações somáticas no protooncogene RET são descritas em apenas $50 \%$ dos casos e parecem não ocorrer de modo uniforme entre as várias subpopulações de células dentro de um mesmo tumor ou de suas metástases (9-13).

Alguns estudos sugerem que o efeito determinado por genes de baixa penetrância possa fornecer uma explicação plausível para essas questões. Seqüências variantes ou polimorfismos genéticos estariam associados a um risco pequeno a moderado para o desenvolvimento da doença.

\section{O QUE SÃO POLIMORFISMOS?}

Dentro de uma espécie, os cromossomos homólogos são bastante similares entre si, mas em determinadas localizações do cromossomo (loci) pode haver variabilidade na seqüência do DNA. Se a variação é encontrada em uma freqüência superior a $1 \%$ da população, denomina-se polimorfismo (14). Polimorfismos podem atuar como marcadores genéticos, já que são transmitidos associados a outros genes localizados na região cromossômica próxima a eles (linkage). Desta forma, se um gene próximo a um marcador causa uma doença, todos os indivíduos afetados na família recebem tanto o marcador como o gene causador da doença (14). Os polimorfismos também são respon- sáveis pela diversidade humana. Diferentes fenótipos são decorrentes de diferentes polimorfismos, como, por exemplo, o sistema ABO (15). De outro modo, os polimorfismos podem influenciar diretamente sobre fatores de risco associados a doenças comuns, como é descrito nos estudos envolvendo a estrutura genética das apolipoproteínas $(16,17)$.

Embora o determinante genético para uma determinada patologia seja identificado através de uma mutação com herança mendeliana simples, os determinantes para a idade de início da doença e a variabilidade clínica existente entre indivíduos com a mesma mutação ainda são ignorados (6-8). Uma explicação possível seria que fatores ambientais, risco ou proteção, uma segunda mutação ou polimorfismo estariam interferindo na evolução da doença (14,18-20).

\section{QUAIS AS BASES GENÉTICAS DO CARCINOMA MEDULAR DE TIREÓIDE?}

O CMT é uma neoplasia maligna com origem nas células $\mathrm{C}$ ou parafoliculares da tireóide, correspondendo a 5 a $8 \%$ dos tumores da glândula tireóide (21). O CMT pode ocorrer na forma esporádica, $75 \%$ dos casos, ou como parte de uma síndrome clínica de herança autossômica dominante (22). Nos pacientes com CMT familiar (CMTF) somente a tireóide é afetada. Os pacientes com neoplasia endócrina múltipla (NEM) 2A desenvolvem CMT, feocromocitoma e/ou hiperparatireoidismo (HPT). Os pacientes com NEM 2B apresentam CMT, feocromocitoma, ganglioneuromas no trato gastrointestinal, neuromas da mucosa com ou sem anormalidades esqueléticas (tabela 1) (23). O líquen amilóide cutâneo (CLA) e a doença de Hirschprung $(\mathrm{DH})$ podem ocorrer associados a MEN 2A (23-29).

O proto-oncogene RET (REarrangement during Transfection) é o gene que determina susce-

Tabela 1. Classificação da NEM 2 de acordo à apresentação clínica.

\begin{tabular}{ll}
\hline NEM 2A & NEM 2A (1): Famílias com CMT, feocromocitoma e hiperparatireoidismo. \\
& $\begin{array}{l}\text { NEM 2A (2): Famílias com CMT, feocromocitoma e sem hiperparatireoidismo. } \\
\text { NEM 2A (3): Famílias com CMT, hiperparatireoidismo e sem feocromocitoma. }\end{array}$ \\
NEM 2B & $\begin{array}{l}\text { Famílias com CMT (com ou sem feocromocitoma), } \\
\text { anormalidades clínicas características e, usualmente, sem hiperparatireoidismo. }\end{array}$ \\
CMTF & $\begin{array}{l}\text { Famílias com no mínimo quatro membros com CMT e sem evidência } \\
\text { de feocromocitoma ou hiperparatireoidismo. }\end{array}$ \\
Outros & $\begin{array}{l}\text { Famílias com menos de quatro indivíduos com CMT e sem evidência de } \\
\text { feocromocitoma ou hiperparatireoidismo. }\end{array}$ \\
\hline
\end{tabular}

Adaptado: International RET Mutation Consortium Analysis; Eng e cols., 1996. 
tibilidade ao CMT (2-4). O conceito de oncogene originou-se com a descoberta de que certos elementos genéticos virais apresentavam a habilidade de formar tumores (30). Diversas classes destes genes foram encontradas em diferentes espécies de vertebrados e invertebrados (30). As sucessivas descobertas levaram rapidamente à observação de que esses genes, quando ativados de modo descontrolado, estariam associados a tumores de origem não-viral em humanos $(2,3)$. Ao contrário dos genes supressores tumorais, os efeitos da ativação de um oncogene nas células são dominantes, ou seja, existe um efeito positivo no crescimento celular mesmo na presença de um alelo normal ou inativado $(30)$.

O proto-oncogene RET está localizado no braço longo do cromossomo 10 (10q11.2) sendo formado por 21 exons $(31,32)$. O RET, expresso nas células derivadas da crista neural, codifica um receptor tirosino-kinase constituído por três domínios, um domínio extracelular com uma região caderina-like e uma região rica em cisteína, um domínio transmembrana e um domínio intracelular tirosino-kinase (5). O receptor RET realiza a transmissão de sinais extracelulares ao núcleo da célula, controlando a proliferação, crescimento e diferenciação celular, sendo ativado pela ligação de um fator de crescimento, denominado glial cell line derived neurotrophic factor (GDNF) (3335). O mecanismo molecular de ativação do RET tem início através da interação do fator de crescimento com um co-receptor glicosil-fosfadidilinositol (GFR $\alpha$ ) ancorado na membrana celular, o qual, por sua vez, se associa ao receptor RET formando um receptor multimérico. A presença do ligante desencadeia a dimerização do RET, ativação do domínio tirosinokinase, determinando assim a transmissão do sinal (figura 1). De outro modo, uma mutação do tipo ganho de função no proto-oncogene RET determina uma ativação constitutiva do receptor RET, ou seja, independente do ligante (5).

Mutações do tipo missense originárias da linhagem germinativa celular são responsáveis pelo CMT hereditário. Os exons mais comumente afetados são o 10,11 e $16(23,27,36-38)$. No entanto, mutações nos exons $5,8,13,14$ e 15 podem ser encontradas mais raramente (39-47). Na imensa maioria dos casos, os pacientes com NEM 2A e CMTF têm a mutação em um de cinco códons (hot points), codificadores dos resíduos de cisteína localizados no domínio extracelular do RET, sendo eles o 609, 611, 618 e 620 (exon 10) e o 634 (exon 11) $(37,38,48-51)$. Nos pacientes com NEM 2B, a mutação mais freqüente afeta $\mathrm{o}$ códon 918 (exon 16), resultando na troca de uma metionina por uma treonina (M918T) no domínio intracelular tirosino-kinase $(4,52)$. No entanto, mutações no códon 883 (exon 15) parecem estar associadas a um pequeno número de casos com NEM 2B (53).

Mutações somáticas no proto-oncogene RET também são descritas em um número variável (23 a 86\%) de casos de CMT esporádico (9-12). Uma mutação somática do tipo missense afetando o códon 918 (exon 16), apresentando a mesma substituição de aminoácidos da NEM 2B, ocorre na maioria dos casos (4). Um estudo realizado em 28 pacientes com CMT esporádico demonstrou que a mutação não ocorre de modo uniforme entre as várias subpopulações de células dentro de um mesmo tumor ou de suas metástases, o que poderia explicar a grande variabilidade na freqüência encontrada na literatura. $\mathrm{O}$ achado também sugere que a mutação no códon 918 pode ter ocorrido durante a evolução clonal de um tumor já estabelecido ou que o mesmo tenha uma origem policlonal (13). Outras mutações, sejam pontuais ou do tipo deleção/ inserção, também foram identificadas em alguns pacientes com CMT esporádico, porém em uma freqüência menor (54-58).

Apesar dos avanços na compreensão da patogênese do CMT, algumas questões permanecem: (1) Por que somente algumas células irão apresentar desenvolvimento clonal se todas são portadoras da mutação no caso do CMT hereditário? (2) Por que indivíduos de uma mesma família apresentam diferença quanto à apresentação clínica, à idade ao diagnóstico e quanto à evolução da doença? (3) Qual o mecanismo genético inicial do CMT esporádico se a mutação somática no códon 918 do proto-oncogene RET for um

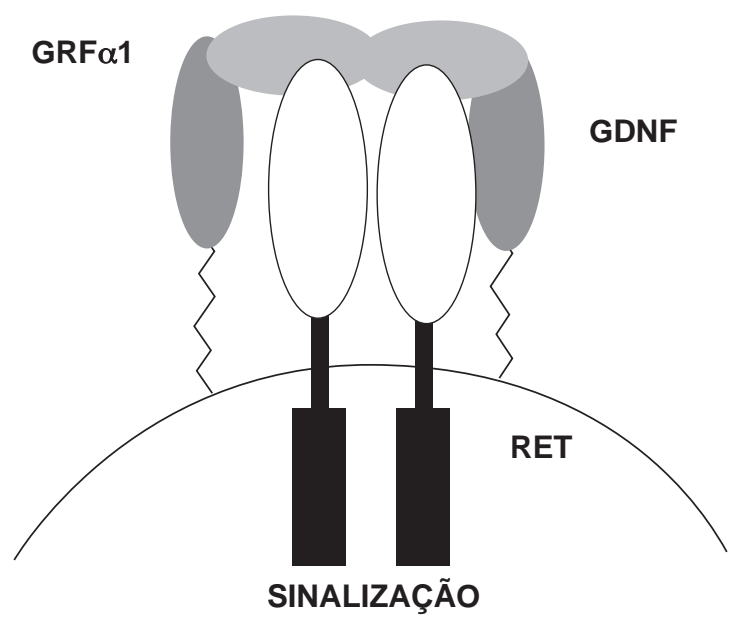

Figura 1. Complexo Multimérico de Ativação do RET. Esquema representando mecanismo de interação do GDNF com as moléculas acessórias GFR $\alpha 1$ promovendo a dimerização e ativação do RET (GDNF: glial cell line derived neurotrophic factor; GFR $\alpha$ 1: GPI-anchored GDNF family receptor $\alpha$ ). 
evento determinado durante a evolução clonal do tumor? (4) Que mecanismo genético explicaria o CMT esporádico entre os pacientes nos quais uma mutação somática no RET não foi identificada?

\section{QUAL O PAPEL DOS POLIMORFISMOS NO CMT?}

Nos últimos anos, diversos autores têm investigado se a presença de seqüências variantes ou polimorfismos poderiam estar associados à susceptibilidade para o desenvolvimento ou modificando a evolução do CMT. A resposta às questões feitas no parágrafo anterior pode ser o efeito determinado por genes de baixa penetrância, ou seja, cuja seqüência variante ou polimorfismo possa estar associado a um risco pequeno a moderado para o desenvolvimento da doença. Desse modo, é razoável supor que polimorfismos, os quais estão presentes numa freqüência relativamente elevada na população, possam conferir um risco atribuível maior do que as raras mutações que ocorrem em genes de susceptibilidade com alta penetrância.

Gimm e cols., analisando 50 casos de CMT esporádico (38 alemães e 12 norte-americanos), observaram um aumento na freqüência do polimorfismo sinônimo no exon 14 , códon $836($ AGC $\rightarrow$ AGT) que codifica uma serina (S836S). A freqüência do alelo S836S foi de $9 \%$ (9/98) entre os casos e 3,7\% (5/140) entre os controles, obtendo na análise uma diferença significativa $(P=0,03)$. Um achado interessante nesse estudo foi que oito dos nove pacientes $(89 \%)$ com o polimorfismo S836S também apresentavam a mutação somática no M918T. Nenhum outro polimorfismo analisado no RET apresentou maior expressão quando associado ao CMT (10).

Em um outro estudo realizado com indivíduos provenientes da região da Andaluzia, na Espanha, Ruiz e cols. analisaram 32 pacientes com CMT esporádico e 250 controles. O polimorfismo S836S apresentou uma freqüência alélica de 9,3\% (6/64) entre os casos comparados a 3,6\% (18/500) dos controles, obtendo-se uma diferença significativa entre os grupos analisados (59).

Elisei e cols. analisaram 106 casos de CMT esporádico, 46 tecidos de pacientes com CMT esporádico, 60 membros de oito famílias com CMT hereditário cujo caso-índice apresentava no exon 11, códon 691, a troca (GGT $\rightarrow$ AGT) de uma glicina por uma serina (G691S), e 106 controles. A análise dos dados demonstrou uma associação positiva entre o polimorfismo G691S e a presença de CMT esporádico. No entanto, não houve diferença nos níveis do RNA mensageiro do RET nos tumores com ou sem o polimorfismo G691S ou com qualquer outro polimorfismo analisado. A presença do polimorfismo G691S foi independente dos achados clínico-patológicos de CMT (estágio TNM, nível de calcitonina e evolução). Outro achado interessante foi a co-segregação da seqüência variante G691S com um ou mais dos polimorfismos neutros. A co-segregação entre o G691S/ S904S (GGT $\rightarrow$ AGT / TCC $\rightarrow$ TCG) foi significativa quando comparada a todas as outras combinações, tanto nos pacientes quanto nos controles. Nos pacientes com CMT hereditário, a transmissão do polimorfismo e da mutação do RET parece ocorrer de forma randômica (60).

Em um outro estudo desenhado para se estimar o risco de CMT hereditário entre pacientes poloneses com diagnóstico de doença aparentemente esporádica, Winch e cols., em 2001, descreveram que o polimorfismo no exon 13, códon 769 (CTT $\rightarrow$ CTG), que codifica uma leucina (L769L), ocorria principalmente em pacientes com CMT esporádico diagnosticado em idades mais precoces (< 30 anos). O polimorfismo apresentou uma freqüência de $36 \%$ nos pacientes com CMT esporádico com idade inferior a 30 anos comparados a $15 \%$ dos que apresentavam entre 31 a 45 anos. O alelo contendo o polimorfismo S836S estava presente em 4,5\% dos pacientes com CMT esporádico. No entanto, a crítica a esse estudo refere-se à ausência de uma população-controle (61).

Robledo e cols. (2003), analisando pacientes com CMT esporádico, observou que o haplótipo G691S/S904S na forma homozigótica foi mais prevalente entre os pacientes com CMT comparados ao grupo controle, sugerindo que a variante tenha um papel como gene de baixa penetrância $(\mathrm{OR}=2,36 ; P=$ $0,045)$. Na análise dos pacientes com CMT hereditário, aqueles que eram homozigotos para o polimorfismo G691S/S904S apresentavam a doença mais precocemente que os pacientes heterozigotos e wildtype. No entanto, em estudo mais recente, 384 indivíduos com NEM 2A pertencentes a 178 famílias foram avaliados quanto ao efeito determinado pelo haplótipo G691S/S904S sobre a idade de aparecimento da doença, e os achados de Robledo e cols. não foram confirmados (62).

Em nosso meio, Magalhães e cols. (47), estudando uma pequena série de pacientes, observaram a ocorrência do polimorfismo CTT por CTG, em heterozigose, no exon 13 (L769L) em 4 pacientes. Observaram também o polimorfismo TCC $\rightarrow$ TCG, em heterozigose, no exon 15 (S904S). Cabe salientar, que uma paciente com o diagnóstico presumido de CMT esporádico apresentava a mutação V804M, no exon 
14, associada ao polimorfismo L769L, no exon 13, o que modificou seu diagnóstico para CMT familiar. A mutação V804M é rara e está associada ao CMTF de aparecimento tardio e menor agressividade. Essa paciente, ao contrário do que usualmente ocorre, teve seu diagnóstico estabelecido aos 32 anos, quando extensas metástases ganglionares foram detectadas. Sua mãe, aos 60 anos, era assintomática e ao ser submetida a rastreamento familiar foram detectados a mutação referida e um tumor de $4 \mathrm{~mm}$, diagnosticado por punção aspirativa com agulha fina. Considerando que a paciente herdou o polimorfismo de seu pai, sua apresentação clínica atípica é consistente com as observações de Wiench e cols. (61), que sugerem que o polimorfismo L769L pode modular a apresentação fenotípica do CMT em indivíduos com predisposição.

No estudo realizado por Cebrian e cols. (2005), os autores também relataram associação positiva entre polimorfismos do RET e o CMT. Um aumento de 1,5 a 2,5 vezes no risco relativo para o desenvolvimento de CMT foi observado entre aqueles que apresentavam os polimorfismos no exon 11 (G691S), exon 15 (S904S) e exon 19 (STOP+388bp). De outro modo, o polimorfismo sinônimo no exon 2 (GCG $\rightarrow$ GCA), que codifica uma alanina (A45A), ocorreu em uma menor freqüência entre os casos de CMT e, segundo Cebrian, poderia representar um alelo protetor contra o desenvolvimento do CMT (63).

Baumgartner-Parzer e cols. (2005) não identificaram diferença quanto à freqüência dos polimorfismos G691S, L769L, S836S e S904S entre pacientes com CMT esporádico, CMTF, indivíduos com calcitonina basal elevada e indivíduos com calcitonina basal normal. Entretanto, um polimorfismo do intron 14 (IVS14-24, seqüência variante distante 24 nucleotídeos do início do exon 15) apresentou uma maior freqüência nos indivíduos com calcitonina basal elevada e com CMT esporádico comparado aos controles (indivíduos com calcitonina basal normal). Entre os pacientes com CMTF foi observada uma associação significativa entre a presença do polimorfismo L769L e uma mutação no códon 791, mutação esta representada pela substituição de uma fenilalanina por uma tirosina (F769Y). Os autores sugerem que a presença do polimorfismo poderia levar à ocorrência da mutação F769Y de novo ou estar modulando o fenótipo da doença; no entanto, pelo número limitado de indivíduos analisados ( 9 casos-índice), as conclusões são, no momento, meramente especulativas (64).

Por outro lado, outros estudos não demonstraram diferenças quanto à presença dos polimorfismos entre pacientes com CMT esporádico e controles.
Berard e cols. analisaram a presença dos polimorfismos L769L e S836S em 92 pacientes com CMT esporádico e 87 controles, todos franceses, não havendo diferença na distribuição dos polimorfismos entre os grupos analisados (65). Na América Latina, Wohllk e cols. estudaram 50 pacientes com tumores esporádicos e 50 controles com etnias semelhantes, predominantemente de origem espanhola, mas a freqüência alélica para os polimorfismos G691S, L769L, S836S e S904S não foi diferente para casos e controles (66).

$\mathrm{O}$ nosso grupo estudou 43 pacientes com CMT esporádico acompanhados no Hospital das Clínicas de Porto Alegre, quanto à presença dos polimorfismos L769L, S836S e S904S, comparados a 62 controles, pareados para sexo e etnia (67). A análise dos resultados não revelou associação significativa entre os polimorfismos L769L, S836S e S904S e a presença de CMT, quando freqüência alélica foi analisada. O polimorfismo L769L foi identificado em 33,6\% dos pacientes CMT esporádico comparado a $24,2 \%$ dos controles, entretanto a diferença encontrada entre os grupos não alcançou significância estatística $(P=0,08)$. O polimorfismo S836S estava presente em $7,0 \%$ dos casos, enquanto $3,2 \%$ dos controles apresentavam o alelo variante S836S $(P=0,32)$. A freqüência encontrada para a variante $\$ 904 S$ foi idêntica entre os grupos, ocorrendo em 22,1\% dos casos e em $21,0 \%$ dos controles $(P=0,86)$. A tabela 2 resume os resultados dos estudos previamente descritos.

Uma possível influência de polimorfismos do RET na doença de Hirschsprung (DH) foi também investigada (68). A DH é uma desordem genética que cursa com obstrução intestinal funcional secundária a aganglionose entérica e que pode estar associada a uma mutação inativadora do RET. Os autores observaram uma maior freqüência dos polimorfismos A45A e L769L comparada à população controle. De outro modo, os polimorfismos G691S e S904S apresentaram uma freqüência menor entre os pacientes com DH comparado aos controles (68).

\section{QUE MECANISMOS SÃO SUGERIDOS?}

O mecanismo preciso que explique como os polimorfismos desempenham seus efeitos sobre o desenvolvimento ou sobre a evolução do CMT não é conhecido. Um dos mecanismos propostos é que o polimorfismo poderia influenciar a estabilidade da molécula do RNA mensageiro (RNAm). No entanto, estudos quantitativos do RNAm do RET no tecido tumoral de indivíduos com CMT, realizados por Elisei e cols. em 2004, não demonstraram diferença na expressão desse gene em pacientes com e sem polimor- 
Tabela 2. Freqüência dos polimorfismos (SNPs) do RET em diferentes populações analisadas.

\begin{tabular}{|c|c|c|c|c|c|c|c|c|c|c|c|c|c|c|c|c|}
\hline \multirow[t]{2}{*}{ SNPs } & \multicolumn{2}{|c|}{$\begin{array}{l}\text { Gimm e cols. } \\
\qquad(n=50)\end{array}$} & \multicolumn{2}{|c|}{$\begin{array}{l}\text { Ruiz e cols. } \\
\qquad(n=32)\end{array}$} & \multicolumn{2}{|c|}{$\begin{array}{l}\text { Berard e cols. } \\
\qquad(n=92)\end{array}$} & \multicolumn{2}{|c|}{$\begin{array}{l}\text { Wohllk } \\
\text { e cols. } \\
(n=50)\end{array}$} & \multicolumn{2}{|c|}{$\begin{array}{l}\text { Elisei e cols. } \\
\qquad(n=106)\end{array}$} & \multicolumn{2}{|c|}{$\begin{array}{l}\text { Cebrian } \\
\text { e cols. } \\
(n=135)\end{array}$} & \multicolumn{2}{|c|}{$\begin{array}{l}\text { Baungartner- } \\
\text { Parze e cols. } \\
\quad(n=45)\end{array}$} & \multicolumn{2}{|c|}{$\begin{array}{l}\text { Rocha e cols. } \\
\qquad(n=43)\end{array}$} \\
\hline & $\begin{array}{c}\text { Caso } \\
(\%)\end{array}$ & $\begin{array}{c}\text { Controle } \\
\text { (\%) }\end{array}$ & $\begin{array}{c}\text { Caso } \\
(\%)\end{array}$ & $\begin{array}{c}\text { Controle } \\
\text { (\%) }\end{array}$ & $\begin{array}{c}\text { Caso } \\
(\%)\end{array}$ & $\begin{array}{c}\text { Controle } \\
\text { (\%) }\end{array}$ & $\begin{array}{c}\text { Caso } \\
(\%)\end{array}$ & $\begin{array}{c}\text { Controle } \\
\text { (\%) }\end{array}$ & $\begin{array}{c}\text { Caso } \\
\text { (\%) }\end{array}$ & $\begin{array}{c}\text { Controle } \\
\text { (\%) }\end{array}$ & $\begin{array}{c}\text { Caso } \\
(\%)\end{array}$ & $\begin{array}{c}\text { Controle } \\
\text { (\%) }\end{array}$ & $\begin{array}{c}\text { Caso } \\
(\%)\end{array}$ & $\begin{array}{c}\text { Controle } \\
\text { (\%) }\end{array}$ & $\begin{array}{c}\text { Caso } \\
(\%)\end{array}$ & $\begin{array}{c}\text { Controle } \\
\text { (\%) }\end{array}$ \\
\hline \multirow[t]{2}{*}{ L769L } & 26,0 & 26,0 & NA & NA & 22,3 & 25,9 & 23,0 & 24,0 & 21,6 & 24,0 & 25,0 & 23,4 & 24,4 & 17,7 & 33,7 & 24,2 \\
\hline & \multicolumn{2}{|c|}{ NS } & & & \multicolumn{2}{|c|}{ NS } & \multicolumn{2}{|c|}{ NS } & \multicolumn{2}{|r|}{ NS } & \multicolumn{2}{|c|}{ NS } & \multicolumn{2}{|c|}{ NS } & \multicolumn{2}{|c|}{ NS } \\
\hline \multirow[t]{2}{*}{ S836S } & 9,0 & 3,7 & 9,3 & 3,6 & 6,5 & 5,2 & 1,0 & 6,0 & 6,1 & 8,4 & 4,4 & 4,6 & 4,4 & 5,7 & 7,0 & 3,2 \\
\hline & \multicolumn{2}{|c|}{$P=0,03$} & \multicolumn{2}{|c|}{$P=0,04$} & \multicolumn{2}{|c|}{ NS } & \multicolumn{2}{|c|}{ NS } & \multicolumn{2}{|c|}{ NS } & \multicolumn{2}{|c|}{ NS } & \multicolumn{2}{|c|}{ NS } & \multicolumn{2}{|c|}{ NS } \\
\hline \multirow[t]{2}{*}{ S904S } & 20,0 & 21,0 & NA & NA & NA & NA & 27,0 & 28,0 & 23,5 & 18,8 & 26,8 & 17,7 & 11,1 & 17,7 & 22,1 & 21,0 \\
\hline & \multicolumn{2}{|c|}{ NS } & & & & & \multicolumn{2}{|c|}{ NS } & \multicolumn{2}{|c|}{ NS } & \multicolumn{2}{|c|}{$P=0,002$} & \multicolumn{2}{|c|}{ NS } & \multicolumn{2}{|c|}{ NS } \\
\hline \multirow[t]{2}{*}{ G691S } & 23,0 & 21,0 & NA & NA & NA & NA & 25,0 & 25,0 & 27,8 & 18,8 & 23,3 & 17,7 & 11,1 & 19,6 & NA & NA \\
\hline & \multicolumn{2}{|c|}{ NS } & & & & & \multicolumn{2}{|c|}{ NS } & & 0,029 & & 0,003 & & NS & & \\
\hline
\end{tabular}

NS, não significativo. NA, não avaliado

fismos (60). Outra hipótese é que a troca de base na molécula do DNA cause a criação de um splicing alternativo levando a uma proteína alterada, ou então que o nucleotídeo modificado esteja em desequilíbrio de ligação com uma variante funcional ainda não conhecida (10). Nos polimorfismos onde ocorre uma substituição de aminoácido, pode-se supor que a modificação tenha um efeito cooperativo na dimerização do RET ou forme um novo sítio de fosforilação no domínio tirosino-kinase (7).

\section{QUAL A EXPLICAÇÃO PARA OS DIFERENTES ACHADOS NOS ESTUDOS?}

Diferenças na etnia podem ser uma das causas para os diferentes resultados descritos. Diferentes atributos genéticos podem conferir proteção ou risco para $o$ desenvolvimento do câncer em populações de ancestralidades diferentes. Porém, estratificar os grupos quanto à etnia não é tão simples, pois nem a cor da pele nem a região de origem podem adequadamente diferenciar uma população miscigenada.

Questões metodológicas também precisam ser discutidas. O CMT é um tumor raro e freqüentemente um número pequeno de pacientes costuma ser analisado. Desta forma, pode-se imaginar que tanto um erro do tipo $\alpha$ (concluir que existe uma associação entre o gene e a doença quando ela não existe) quanto do tipo $\beta$ (concluir que não existe associação quando na verdade existe) podem ser esperados. O problema de interpretação pode ser minimizado se o cálculo do tamanho da amostra estivesse disponível para o leitor. Além disso, um estudo de associação ideal deveria apresentar um número grande de pacientes, um pequeno valor $P$, um risco atribuível alto, uma explicação biológica plausível e pelo menos um outro estudo replicando os achados (69), de modo que somente seria possível analisar um número adequado de pacientes com CMT se houvesse a colaboração conjunta de diversos centros de pesquisa.

\section{AGRADECIMENTOS}

Suporte Financeiro: Conselho Nacional de Desenvolvimento Científico e Tecnológico $(\mathrm{CNPq})$ e Fundação de Amparo à Pesquisa, do Rio Grande do Sul (FAPERGS), Fundação de Amparo à Pesquisa do Estado de São Paulo (FAPESP).

\section{REFERÊNCIAS}

1. Takahashi M, Ritz J, Cooper GM. Activation of a novel human transforming gene, ret, by DNA rearrangement. Cell 1985;42(2):581-8.

2. Mulligan LM, Kwok JB, Healey CS, Elsdon MJ, Eng C, Gardner $E$, et al. Germ-line mutations of the RET proto-oncogene in multiple endocrine neoplasia type 2A. Nature 1993;363(6428):458-60.

3. Donis-Keller H, Dou S, Chi D, Carlson KM, Toshima K, Lairmore TC, et al. Mutations in the RET proto-oncogene are associated with MEN 2A and FMTC. Hum Mol Genet 1993;2(7):851-6.

4. Hofstra RM, Landsvater RM, Ceccherini I, Stulp RP, Stelwagen $T$, Luo $Y$, et al. A mutation in the RET proto-oncogene associated with multiple endocrine neoplasia type $2 \mathrm{~B}$ and sporadic medullary thyroid carcinoma. Nature 1994:367(6461):375-6.

5. Santoro M, Carlomagno F, Melillo RM, Billaud M, Vecchio G, Fusco A. Molecular mechanisms of RET activation in human neoplasia. J Endocrinol Invest 1999;22(10):811-9.

6. Wiench M, Wygoda Z, Gubala E, Wloch J, Oczko M, Jarzab $B$. The genetic background of medullary thyroid carcinoma in young patients. Folia Histochem Cytobiol 2001;39(suppl 2):163-4.

7. Robledo M, Gil L, Pollan M, Cebrián A, Ruíz S, Azañedo M, et al. Polymorphisms G691S/S904S of RET as genetic modifiers of MEN 2A. Cancer Res 2003;63(8):1814-7. 
8. Punales MK, Graf H, Gross JL, Maia AL. RET codon 634 mutations in multiple endocrine neoplasia type 2: variable clinical features and clinical outcome. J Clin Endocrinol Metab 2003;88(6):2644-9.

9. Eng C, Mulligan LM. Mutations of the RET proto-oncogene in the multiple endocrine neoplasia type 2 syndromes, related sporadic tumours, and Hirschsprung disease. Hum Mutat 1997;9(2):97-109.

10. Gimm O, Neuberg DS, Marsh DJ, Dahia PL, Hoang-Vu C Raue $F$, et al. Over-representation of a germline RET sequence variant in patients with sporadic medullary thyroid carcinoma and somatic RET codon 918 mutation. Oncogene 1999;18(6):1369-73.

11. Marsh DJ, Andrew SD, Eng C, Learoyd DL, Capes AG, Pojer $\mathrm{R}$, et al. Somatic mutations in the RET proto-oncogene in sporadic medullary thyroid carcinoma. Clin Endocrinol (Oxf) 1996;44(3):249-57.

12. Eng C, Mulligan LM, Smith DP, Healey CS, Frilling A, Raue F, et al. Mutation of the RET protooncogene in sporadic medullary thyroid carcinoma. Genes Chromosomes Cancer 1995;12(3):209-12

13. Eng C, Mulligan LM, Healey CS, Houghton C, Frilling A, Raue $F$, et al. Heterogeneous mutation of the RET proto-oncogene in subpopulations of medullary thyroid carcinoma. Cancer Res 1996;56(9):2167-70.

14. Balasubramanian SP, Cox A, Brown NJ, Reed MW. Candidate gene polymorphisms in solid cancers. Eur J Surg Oncol 2004;30(6):593-601.

15. Olsson ML, Irshaid NM, Hosseini-Maaf B, Hellberg A, Moulds MK, Sareneva $H$, et al. Genomic analysis of clinical samples with serologic $A B O$ blood grouping discrepancies: identification of 15 novel $A$ and $B$ subgroup alleles. Blood 2001:98(5):1585-93.

16. Kaprio J, Ferrell RE, Kottke BA, Kamboh MI, Sing CF. Effects of polymorphisms in apolipoproteins $E, A-I V$, and $H$ on quantitative traits related to risk for cardiovascular disease. Arterioscler Thromb 1991;11(5):1330-48.

17. Syvanen AC. Accessing genetic variation: genotyping single nucleotide polymorphisms. Nat Rev Genet 2001;2(12):930-42.

18. Alberg AJ, Brock MV, Samet JM. Epidemiology of lung cancer: looking to the future. J Clin Oncol 2005;23(14):3175-85.

19. McGarr SE, Ridlon JM, Hylemon PB. Diet, anaerobic bacterial metabolism, and colon cancer: a review of the literature. $\mathbf{J}$ Clin Gastroenterol 2005;39(2):98-109.

20. Huang SC, Torres-Cruz J, Pack SD, Koch CA, Vortmeyer AO, Mannan $P$, et al. Amplification and overexpression of mutant RET in multiple endocrine neoplasia type 2-associated medullary thyroid carcinoma. J Clin Endocrinol Metab 2003;88(1):459-63.

21. Hazard JB. The C cells (parafollicular cells) of the thyroid gland and medullary thyroid carcinoma. A review. Am J Pathol 1977;88(1):213-50

22. Saad MF, Ordonez NG, Guido JJ, Samaan NA. The prognostic value of calcitonin immunostaining in medullary carcino$\mathrm{ma}$ of the thyroid. J Clin Endocrinol Metab 1984;59(5):850-6.

23. Eng C, Clayton D, Schuffenecker I, Lenoir G, Cote G, Gagel RF et al. The relationship between specific RET proto-oncogene mutations and disease phenotype in multiple endocrine neoplasia type 2. International RET mutation consortium analysis. JAMA 1996;276(19):1575-9.

24. Blank RD, Sklar CA, Dimich AB, LaQuaglia MP, Brennan MF. Clinical presentations and RET protooncogene mutations in seven multiple endocrine neoplasia type 2 kindreds. Cancer 1996;78(9):1996-2003

25. Caron P, Attie T, David D, Amiel J, Brousset F, Roger $P$, et al. C618R mutation in exon 10 of the RET proto-oncogene in a kindred with multiple endocrine neoplasia type $2 \mathrm{~A}$ and Hirschsprung's disease. J Clin Endocrinol Metab 1996;81(7):2731-3

26. Decker RA, Peacock $M L$, Watson $P$. Hirschsprung disease in MEN 2A: increased spectrum of RET exon 10 genotypes and strong genotype-phenotype correlation. Hum Mol Genet 1998;7(1):129-34.
27. Mulligan LM, Marsh DJ, Robinson BG, Schuffenecker I, Zedenius J, Lips CJ, et al. Genotype-phenotype correlation in multiple endocrine neoplasia type 2: report of the International RET Mutation Consortium. J Intern Med 1995;238(4):343-6.

28. Hofstra RM, Sijmons RH, Stelwagen T, Stulp RP, Kousseff BG, Lips $\mathrm{CJ}$, et al. RET mutation screening in familial cutaneous lichen amyloidosis and in skin amyloidosis associated with multiple endocrine neoplasia. J Invest Dermatol 1996;107(2):215-8.

29. Punales MK, Rocha AP, Gross JL, Maia AL. [Medullary thyroid carcinoma: clinical and oncological features and treatment]. Arq Bras Endocrinol Metab 2004;48(1):137-46.

30. Krontiris TG. Oncogenes. N Engl J Med 1995;333(5):303-6.

31. Ishizaka Y, Itoh F, Tahira T, Ikeda I, Sugimura T, Tucker J, et al. Human ret proto-oncogene mapped to chromosome 10q11.2. Oncogene 1989;4(12):1519-21

32. Pasini B, Hofstra RM, Yin L, Bocciardi R, Santamaria G, Grootscholten PM, et al. The physical map of the human RET proto-oncogene. Oncogene 1995;11(9):1737-43.

33. Ponder BA, Smith D. The MEN II syndromes and the role of the ret proto-oncogene. Adv Cancer Res 1996;70:179-222.

34. Eng C. RET proto-oncogene in the development of human cancer. J Clin Oncol 1999;17(1):380-93

35. Saarma M. GDNF - a stranger in the TGF- $\beta$ superfamily? Eur J Biochem 2000;267(24):6968-71.

36. Maruyama S, Iwashita T, Imai T, Funahashi H, Ceccherini I, Luo $Y$, et al. Germ line mutations of the ret proto-oncogene in Japanese patients with multiple endocrine neoplasia type $2 \mathrm{~A}$ and type 2B. Jpn J Cancer Res 1994;85(9):879-82.

37. Mulligan LM, Eng C, Healey CS, Clayton D, Kwok JB, Gardner E, et al. Specific mutations of the RET proto-oncogene are related to disease phenotype in MEN 2A and FMTC. Nat Genet 1994;6(1):70-4.

38. Heshmati HM, Gharib $\mathrm{H}$, Khosla $\mathrm{S}$, Abu-Lebdeh HS, Lindor NM, Thibodeau SN. Genetic testing in medullary thyroid carcinoma syndromes: mutation types and clinical significance. Mayo Clin Proc 1997:72(5):430-6.

39. Dvorakova S, Vaclavikova E, Duskova J, Vlcek P, Ryska A, Bendlova B. Exon 5 of the RET proto-oncogene: a newly detected risk exon for familial medullary thyroid carcinoma, a novel germ-line mutation Gly321Arg. J Endocrinol Invest 2005;28(10):905-9.

40. Hofstra RM, Fattoruso O, Quadro L, Wu Y, Libroia A, Verga U, et al. A novel point mutation in the intracellular domain of the ret protooncogene in a family with medullary thyroid carcinoma. J Clin Endocrinol Metab 1997;82(12):4176-8.

41. Elisei R, Cosci B, Romei C, Agate L, Piampiani P, Miccoli P, et al. Identification of a novel point mutation in the RET gene (Ala883Thr), which is associated with medullary thyroid carcinoma phenotype only in homozygous condition. J Clin Endocrinol Metab 2004:89(11):5823-7.

42. Bolino A, Schuffenecker I, Luo Y, Seri M, Silengo M, Tocco T, et al. RET mutations in exons 13 and 14 of FMTC patients. Oncogene 1995; 10(12):2415-9.

43. Feldman GL, Edmonds MW, Ainsworth PJ, Schuffenecker I, Lenoir GM, Saxe AW, et al. Variable expressivity of familial medullary thyroid carcinoma (FMTC) due to a RET V804M (GTG-->ATG) mutation. Surgery 2000;128(1):93-8.

44. Wohllk N, Becker P, Youlton R, Cote GJ, Gagel RF. [Germline mutations of the ret proto-oncogene in Chilean patients with hereditary and sporadic medullary thyroid carcinoma]. Rev Med Chil 2001;129(7):713-8.

45. Gimm O, Niederle BE, Weber T, Bockhorn M, Ukkat J, Brauckhoff $M$, et al. RET proto-oncogene mutations affecting codon 790/791: A mild form of multiple endocrine neoplasia type 2A syndrome? Surgery 2002;132(6):952-9; discussion 9.

46. Berndt I, Reuter M, Saller B, Frank-Raue K, Groth P, Grussendorf $M$, et al. A new hot spot for mutations in the ret protooncogene causing familial medullary thyroid carcinoma and multiple endocrine neoplasia type 2A. J Clin Endocrinol Metab 1998;83(3):770-4.

47. Magalhães PK, de Castro M, Elias LL, Soares EG, Maciel LM. Polymorphisms in the RET proto-oncogene and the phenotypic presentation of familial medullary thyroid carcinoma. Thyroid 2004;14(10):848-52. 
48. Mulligan LM, Eng C, Attie T, Lyonnet S, Marsh DJ, Hyland VJ, et al. Diverse phenotypes associated with exon 10 mutations of the RET proto-oncogene. Hum Mol Genet 1994;3(12):2163-7.

49. Oriola J, Paramo C, Halperin I, Garcia-Mayor RV, Rivera-Fillat F. Novel point mutation in exon 10 of the RET proto-oncogene in a family with medullary thyroid carcinoma. Am $\mathbf{J}$ Med Genet 1998;78(3):271-3.

50. Marsh DJ, Robinson BG, Andrew S, Richardson AL, Pojer R, Schnitzler M, et al. A rapid screening method for the detection of mutations in the RET proto-oncogene in multiple endocrine neoplasia type $2 \mathrm{~A}$ and familial medullary thyroid carcinoma families. Genomics 1994:23(2):477-9.

51. Komminoth P, Kunz EK, Matias-Guiu X, Hiort O, Christiansen G, Colomer A, et al. Analysis of RET protooncogene point mutations distinguishes heritable from nonheritable medullary thyroid carcinomas. Cancer 1995;76(3):479-89.

52. Eng C, Smith DP, Mulligan LM, Nagai MA, Healey CS, Ponder $M A$, et al. Point mutation within the tyrosine kinase domain of the RET proto-oncogene in multiple endocrine neoplasia type $2 B$ and related sporadic tumours. Hum Mol Genet 1994;3(2):237-41.

53. Gimm O, Marsh DJ, Andrew SD, Frilling A, Dahia PL, Mulligan LM, et al. Germline dinucleotide mutation in codon 883 of the RET proto-oncogene in multiple endocrine neoplasia type 2B without codon 918 mutation. J Clin Endocrinol Metab 1997;82(11):3902-4

54. Hofstra RM, Stelwagen T, Stulp RP, de Jong D, Hulsbeek M, Kamsteeg EJ, et al. Extensive mutation scanning of RET in sporadic medullary thyroid carcinoma and of RET and VHL in sporadic pheochromocytoma reveals involvement of these genes in only a minority of cases. J Clin Endocrinol Metab 1996;81(8):2881-4.

55. Uchino S, Noguchi S, Yamashita H, Sato M, Adachi M, Yamashita $\mathrm{H}$, et al. Somatic mutations in RET exons 12 and 15 in sporadic medullary thyroid carcinomas: different spectrum of mutations in sporadic type from hereditary type. Jpn J Cancer Res 1999;90(11):1231-7.

56. Bugalho MJ, Coelho I, Sobrinho LG. Somatic trinucleotide change encompassing codons 882 and 883 of the RET protooncogene in a patient with sporadic medullary thyroid carcinoma. Eur J Endocrinol 2000;142(6):573-5.

57. Gimm O, Greco A, Hoang-Vu C, Dralle H, Pierotti MA, Eng C. Mutation analysis reveals novel sequence variants in NTRK1 in sporadic human medullary thyroid carcinoma. J Clin Endocrinol Metab 1999;84(8):2784-7.

58. Romei C, Elisei R, Pinchera A, Ceccherini I, Molinaro E, Mancusi $F$, et al. Somatic mutations of the ret protooncogene in sporadic medullary thyroid carcinoma are not restricted to exon 16 and are associated with tumor recurrence. J Clin Endocrinol Metab 1996;81(4):1619-22.

59. Ruiz A, Antinolo G, Fernandez RM, Eng C, Marcos I, Borrego $\mathrm{S}$. Germline sequence variant S836S in the RET proto-oncogene is associated with low level predisposition to sporadic medullary thyroid carcinoma in the Spanish population. Clin Endocrinol (Oxf) 2001;55(3):399-402.
60. Elisei R, Cosci B, Romei C, Bottici V, Sculli M, Lari R, et al. RET exon 11 (G691S) polymorphism is significantly more frequent in sporadic medullary thyroid carcinoma than in the general population. J Clin Endocrinol Metab 2004;89(7):3579-84.

61. Wiench M, Wygoda Z, Gubala E, Wloch J, Lisowska K, Krassowski J, et al. Estimation of risk of inherited medullary thyroid carcinoma in apparent sporadic patients. J Clin Oncol 2001;19(5):1374-80.

62. Lesueur $F$, Cebrian $A$, Robledo $M$, Niccoli-Sire $P$, Svensson $\mathrm{KA}$, Pinson S, et al. Polymorphisms in RET and its coreceptors and ligands as genetic modifiers of multiple endocrine neoplasia type 2A. Cancer Res 2006;66(2):1177-80.

63. Cebrian A, Lesueur F, Martin S, Leyland J, Ahmed S, Luccarini C, et al. Polymorphisms in the initiators of RET (rearranged during transfection) signaling pathway and susceptibility to sporadic medullary thyroid carcinoma. J Clin Endocrinol Metab 2005;90(11):6268-74.

64. Baumgartner-Parzer SM, Lang R, Wagner L, Heinze G, Niederle $\mathrm{B}$, Kaserer $\mathrm{K}$, et al. Polymorphisms in exon 13 and intron 14 of the RET protooncogene: genetic modifiers of medullary thyroid carcinoma? J Clin Endocrinol Metab 2005;90(11):6232-6.

65. Berard I, Kraimps JL, Savagner F, Murat A, Renaudin K, Nicolli-Sire P, et al. Germline-sequence variants S836S and L769L in the RE arranged during Transfection (RET) proto-oncogene are not associated with predisposition to sporadic medullary carcinoma in the French population. Clin Genet 2004;65(2):150-2.

66. Wohllk GN, Soto CE, Bravo AM, Becker CP. [G691S, L769L and S836S ret proto-oncogene polymorphisms are not associated with higher risk to sporadic medullary thyroid carcinoma in Chilean patients]. Rev Med Chil 2005;133(4):397-402.

67. Rocha AP, Puñales MK, Meotti C, Gross JL, Maia AL. Role of RET genetic variants in sporadic and hereditary forms of medullary thyroid carcinoma. (submitted).

68. Borrego S, Saez ME, Ruiz A, Gimm O, Lopez-Alonso M, Antinolo $\mathrm{G}$, et al. Specific polymorphisms in the RET proto-oncogene are over-represented in patients with Hirschsprung disease and may represent loci modifying phenotypic expression. J Med Genet 1999;36(10):771-4.

69. Freely associating. Nat Genet 1999;22(1):1-2.

Endereço para correspondência:

Ana Luiza Maia

Serviço de Endocrinologia

Hospital de Clínicas de Porto Alegre

Rua Ramiro Barcelos 2350

90035-003 Porto Alegre, RS

Fax: (51) 3332-5188

E-mail: almaia@ufrgs.br 